\title{
Use of AUDIT-C alcohol screening tool in NHS general dental practices
}

Renato Venturelli ${ }^{1}$, Antiopi Ntouva ${ }^{1}$, Jessie Porter ${ }^{1}$, Michelle Stennett ${ }^{1}$, Mike J Crawford ${ }^{2}$, Annie Britton ${ }^{1}$, Christine Gratus ${ }^{1}$, Georgios Tsakos ${ }^{1}$, Anja Heilmann ${ }^{1}$, Tim Newton ${ }^{3}$, Caillin Redican ${ }^{4}$, Hynek Pikhart ${ }^{1}$, Richard G Watt ${ }^{1 *}$

${ }^{1}$ Department of Epidemiology and Public Health, UCL, UK. ${ }^{2}$ Department of Medicine, Imperial College London, UK. ${ }^{3}$ King's College London Dental Institute, Kings College London, UK. ${ }^{4}$ General dental practitioner and Health Education England.

* Corresponding author. Department of Epidemiology and Public Health, 1-19 Torrington Place, UCL, London WC1E 6BT, UK. Tel. +44 207679 1699: Fax. + 44207813 0280: Email: r.watt@ucl.ac.uk 


\begin{abstract}
:
Background: The numerous health risks of excessive alcohol consumption are well documented. Individuals at risk of harm from alcohol consumption can be identified through alcohol screening tools; however, there is limited research regarding their use in general dental practices.
\end{abstract}

Methods: Data was collected as part of a feasibility trial evaluating the delivery of brief alcohol advice in general dental practices in North London. Patient demographics and health related behaviours were collected, and the Alcohol Use Disorders Identification Test of Consumption (AUDIT-C) tool was used to assess alcohol consumption patterns.

Results: The analytical sample comprised of 552 dental patients, of whom approximately half $(46 \%)$ were drinking alcohol at hazardous levels. Males, younger adults, those who consumed red meat weekly and smokers all had significantly increased risks of excessive alcohol consumption. Smokers were more likely to consume excessive levels of alcohol irrespective of smoking frequency. Notable sex differences in alcohol consumption were identified, with males being more likely to consume alcohol frequently and in larger quantities than females.

Conclusion: The AUDIT-C tool can be used in general dental practice to screen for harmful levels of alcohol consumption. Clear associations exist between patient demographics, health behaviours and excessive alcohol consumption.

\title{
In brief points:
}

- Brief alcohol screening tools may be employed routinely in general dental practice to identify patients at risk of harm from excessive alcohol consumption

- Harmful levels of alcohol consumption were identified in nearly half of the patients screened

- Males, younger adults, smokers and those who consumed red meat weekly were more likely to consume levels of alcohol above the recommended limits 


\section{Background}

Excessive alcohol consumption has a significant disease, social and economic burden across the population. ${ }^{1,2}$ Drinking alcohol is linked with a risk of developing a range of physical and mental health problems and is a causal risk factor in more than 200 conditions. ${ }^{1,3}$ The higher the amount and frequency of alcohol consumption, the higher the risks of alcohol-related harm. ${ }^{1,4}$ Alcohol consumption can have an effect not only on the incidence of diseases, but also on the long term prognosis of health conditions and their outcomes. ${ }^{5}$ In England, there were 1.2 million hospital admissions linked to alcohol consumption in $2017 / 18$, with $61 \%$ of them being for males and 39\% for females. ${ }^{6}$ Moreover, there were 5,698 deaths directly attributed to alcohol consumption in $2018 .{ }^{7}$ Consuming alcohol also increases the risk of a number of oral conditions, including oral and oropharyngeal cancers, as well as facial injuries and dental trauma. ${ }^{8,9}$ Research has also shown that alcohol misuse is associated with periodontal disease, dental erosion, halitosis and tooth staining. ${ }^{10-14}$

The current UK Chief Medical Officers' Low Risk Drinking Guidelines state that it is safest not to drink more than 14 units of alcohol a week on a regular basis to keep health risks at a low level. For those who consume alcohol up to this amount, it is advised that drinking should be spread over three or more days. ${ }^{15}$ Findings from the 2018 Health Survey for England (HSE) showed that $82 \%$ of adults had drunk alcohol in the previous twelve months, with a higher proportion of males than females drinking over 14 units per week (30\% and $14 \%$, respectively). HSE 2018 also revealed that the proportion of adults drinking above recommendations was most common among those aged 55 to 64 years (38\% of males and $19 \%$ of females). ${ }^{16}$

Identifying individuals who are drinking above recommended levels is important, as the majority of drinkers are unaware that they are not complying with guidance limits. ${ }^{17}$ In line with the aforementioned recommendations and with those made by the National Institute of Health and Care Excellence (NICE) about screening individuals for alcohol misuse in health and social care settings, ${ }^{17}$ a number of alcohol screening tools have been developed and validated. Screening tools for use in primary care include: the Fast Alcohol Screening Test $\left(\right.$ FAST) ${ }^{18}$; the Modified-Single Alcohol Screening Question (M-SASQ) ${ }^{19}$; the Alcohol Use Disorders Identification Test (AUDIT) ${ }^{20}$; and an abbreviated version of AUDIT containing its first three questions: the Alcohol Use Disorders Identification Test of 
Consumption (AUDIT-C). ${ }^{18-21}$ This shortened version of the AUDIT has been advocated for use in both research and primary care settings where time may be limited, as it has been shown to have similar accuracy to the full AUDIT questionnaire..$^{22,23}$

Due to the direct link between alcohol intake and oral health, and the fact that a relatively high proportion of the general population attend a dentist, ${ }^{24}$ NHS general dental practices may be an ideal setting to identify those who are at higher risk of developing alcohol-related problems. ${ }^{25}$ Goodall and colleagues ${ }^{26}$ in Scotland used the full AUDIT tool to screen 400 patients attending four general dental practices for alcohol misuse and found that 95 patients $(30.8 \%)$ were consuming alcohol at a hazardous level. In Bradford, England, a small feasibility study that used the AUDIT-C tool showed that among a sample of 29 patients attending one general dental practice, only 7 were consuming alcohol within the recommended levels. ${ }^{27}$ In Wales, a small-scale randomised controlled trial that recruited patients aged 18 to 45 years from a general dental practice showed that 46 out of 106 patients $(43 \%)$ were consuming excessive levels of alcohol. ${ }^{28}$

As very little research has been conducted on the use of alcohol screening tools in dental primary care settings in the UK, the objectives of this study were: 1 . To determine the level of alcohol consumption, measured through the AUDIT-C tool, among a sample of patients attending NHS general dental practices in North London; 2. To assess the associations between AUDIT-C scores and patient demographic and other behavioural factors (diet, smoking).

\section{Methods}

This study analysed screening data gathered as part of the Dental Alcohol Reduction Trial (DART; Trial Registration Number: ISRCTN81193263), a two-arm cluster randomised control feasibility trial that evaluated the acceptability and practicality of delivering alcohol brief advice in primary dental care settings. ${ }^{29}$ Ten NHS dental practices from across North London (Islington, Camden, Haringey, Brent, Enfield and Redbridge) participated in the trial.

Adult patients attending the enrolled dental practices were initially opportunistically approached by dental staff to explain the nature of the study. Once verbal consent was obtained, potential participants then completed a screening questionnaire whilst waiting for their dental appointment. Screening data collection took place between May and December 
2014. Ethical approval for the DART study was obtained from the Camden and Islington Research Ethics Committee (Reference: 13/LO/0292).

Alcohol screening data were obtained from the three-item AUDIT-C tool. ${ }^{21,23}$ Initial exploratory work in the DART study involved interviewing dental staff and patients to determine their views on the suitability of screening for alcohol problems in dental practice. ${ }^{29}$ These initial interviews demonstrated that both dental professionals and patients considered AUDIT-C as the preferred instrument to collect screening data. The AUDIT-C questions and scoring system are shown below (Table 1):

Table 1 here

AUDIT-C scores range from 0 to 12 points, with higher scores representing greater levels of alcohol intake. Dental patients who obtained a score of five or more were identified as having drinking levels higher than the recommended safe levels. ${ }^{15}$ Therefore, patients were divided into two groups: those with an AUDIT-C positive score ( $\geq 5$ points) and those with a negative overall score ( $<5$ points).

To mask the focus on alcohol to potential participants, the screening questionnaire also included questions on demographics (age and gender) and on diet and smoking. Participants were asked "How often do you eat five or more servings of fruit or vegetables in one day?" as well as "How often do you eat a serving of red meat (beef, lamb or pork) in one day?". The categories included for both of these variables were: 'never', 'less than once a month', 'once a month', 'weekly', and 'daily'. To measure smoking status two questions were used: “Do you smoke?" and "How often do you smoke cigarettes?" Participants were categorised as 'current smoker', 'ex-smoker', and 'never smoked' in response to the first question. Those who were current smokers were then asked the second question and were classified as either 'less than daily' or 'daily'.

\section{Statistical methods}

Descriptive statistics were employed to describe the demographic characteristics and healthrelated behaviours of the study participants. To assess the association between the overall AUDIT-C score (positive vs negative) and the variables of interest, chi-squared and chisquared-for-trend tests were carried out. In addition, we used bar graphs to present the 
distribution of the three separate AUDIT-C items according to sex. Data analysis was carried out on complete cases using the statistical software package STATA/IC version 15 (StataCorp, College Station, TX). A p-value of $\leq 0.05$ was considered statistically significant.

\section{Results}

In total, 567 dental patients were screened across ten NHS general dental practices in North London. Only 15 participants had missing data and were excluded from the analysis. The final sample size was 552.

Table 2 presents the sample characteristics. Approximately 58\% of the individuals were females. Participants' ages ranged from 18 to 92 years $(M=45.2, S D=15.8)$. Most participants reported consuming five or more servings of fruit or vegetables on a daily $(48.4 \%)$ or weekly $(43.5 \%)$ basis. Around $63 \%$ of the sample reported having red meat weekly, whilst nearly $9 \%$ reported never eating red meat. Regarding smoking, approximately $50 \%$ of the individuals declared they had never smoked and $25 \%$ classified themselves as exsmokers. Of those who were current smokers (24.3\%), most reported smoking on a daily basis (84.2\%). Almost half of the participants (45.8\%) were identified as drinking alcohol above the recommended levels.

\section{Table 2 here}

Figures 1-3 present the breakdown of the individual AUDIT-C items by sex. In relation to the AUDIT-C question on frequency of consumption, approximately $29 \%(n=159)$ of the participants declared that they had an alcoholic drink two to three times per week, and 17\% $(n=93)$ reported consuming alcoholic drinks four and more times per week. As shown in Figure 1, males were more likely to be classified in the more frequent alcohol consumption categories than females $(p<0.001)$. In terms of number of units of alcohol consumed, the results showed that around $16 \%$ of participants reported drinking five to six alcohol units on a typical day when drinking $(n=87)$, and over $3 \%$ stated drinking 10 or more $(n=18)$. It can be seen from the data in Figure 2, that a lower proportion of females than males reported drinking more units of alcohol on a typical day $(\mathrm{p}<0.001)$. Finally, in terms of binge drinking on a single occasion ( 6 or more units if female, or 8 or more if male) most individuals reported never drinking up to this amount $(\mathrm{n}=190 ; 35 \%)$ or doing so less than monthly 
$(n=168 ; 31 \%)$. Significantly fewer females than males reported drinking at or above this level monthly or weekly (42\% vs $62 \%$ and $31 \%$ vs $43 \%$, respectively) as shown in figure 3 .

\section{Figures 1, 2 and 3 here}

The results assessing the association between the overall AUDIT-C scores and the demographic and behavioural factors are shown in Table 3. Females were significantly less likely to have a positive AUDIT-C score ( $\geq 5$ points) than males (OR=0.4; 95\%CI: 0.3-0.5). Individuals aged between 35 and 49 years, as well as those aged 50 years and above were less likely to report drinking at a hazardous level compared to participants in the younger age group (OR=0.5; 95\%CI: 0.4-0.9 and OR=0.3; 95\%CI:0.2-0.5, respectively). Compared to those who reported never eating red meat, those who consumed it weekly were significantly more likely to have a positive overall AUDIT-C score (OR=2.1; 95\%CI:1.1-4.0). Participants who never smoked were significantly less likely to consume alcohol above the recommended levels compared to current smokers $(\mathrm{OR}=0.5$; 95\%CI: $0.3-0.8)$. The proportion of participants who were classified as having a positive AUDIT-C score did not differ by fruit and vegetable consumption and smoking frequency.

\section{Table 3 here}

\section{Discussion}

Our study findings indicate high levels of excessive alcohol consumption among adults attending general dental practices in North London. Nearly half (46\%) of the patients screened in this study scored positive in the AUDIT-C tool, which equates to consuming alcohol above the current recommended safe levels. Within this sample of patients, males, younger adults aged 18-34, those consuming meat weekly and smokers all had a significantly increased risk of scoring AUDIT-C positive. In comparison to females, males consumed alcohol more frequently, were more likely to consume larger quantities of alcohol, and more likely to report consuming higher quantities of alcohol on any single occasion. The consumption of fruit and vegetables and the frequency of smoking among current smokers were not associated with AUDIT-C positive scores.

There are a limited number of similar studies which have used alcohol screening tools in dental practice settings within the UK. The most comparable small scale study $(n=29)$ in a 
single general dental practice in Bradford found that $77 \%$ of patients had AUDIT-C positive scores. ${ }^{27}$ However, another study reported similar levels of excessive drinking to those observed in this study (43\%), using the M-SASQ tool. ${ }^{28}$ Similarly, a study which utilised the full AUDIT tool found that nearly $31 \%$ of adults consumed alcohol at hazardous levels. ${ }^{26}$

When comparing our results with national data from $2018 \mathrm{HSE}$, there are a number of points to highlight. A higher proportion of adults in our sample were drinking alcohol at harmful levels than in the general population (46\% vs $40 \%) .{ }^{16}$ Only $10 \%$ of our participants reported never drinking alcohol, whilst $18 \%$ of adults from England reported not consuming alcohol in the last 12 months. ${ }^{16}$ In relation to differences by sex, our findings were aligned with national estimates that indicate higher levels of hazardous drinking in males compared to females, and this was true for any given measure assessed. Surprisingly, our results contrast those from 2018 HSE in terms of differences by age. The proportion of individuals in our study with positive AUDIT-C scores was highest among those aged 18-34 years, whereas the proportion of adults drinking above recommended levels in the general population was highest among those aged 55-64 years. ${ }^{16}$

Further important points to note are the substantial proportion of individuals who reported being current smokers in our sample (25\%) - considerably higher than the prevalence seen in the general population $(17 \%)^{16}$, and the fact that current smokers were significantly more likely to have positive AUDIT-C scores than those who never smoked, irrespective of whether they smoked daily or less often. There is evidence that health behaviours which increase the risk of chronic disease and mortality (e.g. alcohol consumption, smoking, low fruit and vegetable consumption, and physical inactivity) are not isolated and separated from one another, but tend to occur in clusters. ${ }^{30-32}$ The consumption of alcohol in combination with smoking leads to a synergistic greatly increased risk of oral and oropharyngeal cancers. ${ }^{33}$ The routine use of alcohol screening tools such as AUDIT-C in combination with taking a smoking history may help to identify those patients most at risk, in order to provide appropriate preventive health advice.

In contrast with previous studies that have assessed alcohol consumption within general dental practice settings, this study had a larger sample size of 552 patients from across ten NHS dental practices. The inclusion of demographic information and data related to other health behaviours has enabled this study to identify risk factors in relation to hazardous 
drinking. However, there were no available data relating to the socioeconomic position of patients. The non-random opportunistic approach used to recruit patients may have resulted in selection bias towards patients suspected of consuming alcohol, rather than a representative sample of the dental patient population. Moreover, as the data were only collected from dental practices in North London, the findings are not generalisable across the UK. In addition, some of the categories of the AUDIT-C tool contained very small numbers of responses, which may have affected the validity of the comparisons made.

Future research into the use of alcohol screening tools within general dental practice settings could include a more randomly selected, representative sample and additional demographic data such as socioeconomic position. Further evidence from randomised controlled trials would also help to determine the effectiveness of brief alcohol advice offered in NHS general dental practices.

In conclusion, the AUDIT-C tool can be utilised as a screening tool in general dental practice to identify hazardous drinking behaviours and target the delivery of brief alcohol advice. This study showed that males, younger adults (aged 18-34), and individuals who smoked and consumed red meat weekly were significantly more likely to consume harmful levels of alcohol than their counterparts. However, more largescale research is needed to fully understand and further assess demographic and behavioural factors associated with excessive alcohol consumption, and the effectiveness of brief alcohol advice delivered within general dental practice settings. 


\section{Declaration of interests}

The authors declare no conflict of interests. This paper presents independent research funded by the National Institute for Health Research (NIHR) under its Research for Patient Benefit Programme (Grant Reference Number PB-PG-0212-27029). The views expressed are those of the authors and not necessarily those of the NHS, the NIHR or the Department of Health and Social Care.

\section{Acknowledgements}

The authors would like to express their gratitude to all the dental staff and patients for their cooperation and participation in the study. 


\section{Reference list}

1. World Health Organization. Global status report on alcohol and health 2018. Geneva: World Health Organization. 2018.

2. Public Health England. The Public Health Burden of Alcohol and the Effectiveness and Cost-Effectiveness of Alcohol Control Policies: An evidence review. London: Public Health England. 2016

3. World Health Organization. Global status report on alcohol and health 2014. Geneva: World Health Organization. 2014.

4. Griswold MG, Fullman N, Hawley C, Arian N, Zimsen SRM, Tymeson HD, et al. Alcohol use and burden for 195 countries and territories, 1990-2016: A systematic analysis for the Global Burden of Disease Study 2016. Lancet 2018; 392(10152):1015-35.

5. Shield KD, Parry C, Rehm J. Chronic diseases and conditions related to alcohol use. Alcohol Res Curr Rev 2014; 35(2):155-71.

6. NHS Digital. Part 1: Alcohol-related hospital admissions - NHS Digital. 2019. Online information available at https://digital.nhs.uk/data-andinformation/publications/statistical/statistics-on-alcohol/2019/part-1(accessed March 2020].

7. NHS Digital. Part 2: Alcohol-specific deaths - NHS Digital. 2020. Online information available at https://digital.nhs.uk/data-andinformation/publications/statistical/statistics-on-alcohol/2020/part-2 (accessed March 2020).

8. Turati F, Garavello W, Tramacere I, Pelucchi C, Galeone C, Bagnardi V, et al. A Meta-analysis of Alcohol Drinking and Oral and Pharyngeal Cancers: Results from Subgroup Analyses. Alcohol Alcohol 2013; 48(1):107-18.

9. Hutchison IL, Magennis P, Shepherd JP, Brown AE. The BAOMS United Kingdom survey of facial injuries part 1: aetiology and the association with alcohol consumption. British Association of Oral and Maxillofacial Surgeons. Br J Oral Maxillofac Surg 1998; 36(1):3-13.

10. Amaral C da SF, Vettore MV, Leão A. The relationship of alcohol dependence and alcohol consumption with periodontitis: A systematic review. J Dent 2009; 37(9):64351.

11. Rosenberg M, Knaan T, Cohen D. Association among Bad Breath, Body Mass Index, 
and Alcohol Intake. J Dent Res 2007; 86(10):997-1000.

12. Robb ND, Smith BG. Prevalence of pathological tooth wear in patients with chronic alcoholism. Br Dent J 1990; 169(11):367-9.

13. Suwama K, Yoshihara A, Watanabe R, Stegaroiu R, Shibata S, Miyazaki H. Relationship between alcohol consumption and periodontal tissue condition in community-dwelling elderly Japanese. Gerodontology 2018; 35(3):170-6.

14. Pitiphat W, Merchant AT, Rimm EB, Joshipura KJ. Alcohol consumption increases periodontitis risk. J Dent Res 2003; 82(7):509-13.

15. Department of Health. UK Chief Medical Officers' Low Risk Drinking Guidelines. London: Department of Health. 2016.

16. Population Health Team, NHS Digital. Health Survey for England 2018: Adult heath related behaviours. London: NHS Digital. 2019.

17. National Institute for Health and Clinical Excellence (NICE). Alcohol-use disorders: diagnosis, assessment and management of harmful drinking (high-risk drinking) and alcohol dependence. Clinical guideline (CG115). London: NICE. 2011.

18. Hodgson R, Alwyn T, John B, Thom B, Smith A. The FAST Alcohol Screening Test. Alcohol Alcohol 2002; 37(1):61-6.

19. Screening and Intervention Programme for Sensible drinking (SIPS). Modified-Single Alcohol Screening Question (M-SAS). 2008. Online information available at https://www.kcl.ac.uk/research/sips (accessed April 2020).

20. Saunders JB, Aasland OG, Babor TF, de la Fuente JR, Grant M. Development of the Alcohol Use Disorders Identification Test (AUDIT): WHO Collaborative Project on Early Detection of Persons with Harmful Alcohol Consumption--II. Addiction 1993; 88(6):791-804.

21. Bush K, Kivlahan DR, McDonell MB, Fihn SD, Bradley KA. The AUDIT alcohol consumption questions (AUDIT-C): An effective brief screening test for problem drinking. Arch Intern Med 1998; 158(16):1789-95.

22. National Institute for Health and Care Excellence (NICE). Alcohol-use disorders: prevention. London: NICE. 2010.

23. Bradley KA, Debenedetti AF, Volk RJ, Williams EC, Frank D, Kivlahan DR. AUDITC as a brief screen for alcohol misuse in primary care. Alcohol Clin Exp Res 2007; 31(7):1208-17.

24. NHS Dental Statistics. NHS Dental Statistics for England 2019-20, Biannual Report NHS Digital. 2020. Online information available at https://digital.nhs.uk/data-and- 
information/publications/statistical/nhs-dental-statistics/2019-20-biannual-report (accessed April 2020).

25. McAuley A, Goodall CA, Ogden GR, Shepherd S, Cruikshank K. Delivering alcohol screening and alcohol brief interventions within general dental practice: rationale and overview of the evidence. Br Dent J 201; 210(9):E15-E15.

26. Goodall C., Crawford A, Macaskill S, Welbury R. Assessment of hazardous drinking in General Dental Practice. J Dent Res 2006; 85(B:1219).

27. Csikar J, Paige C, Godson J. The feasibility of using an alcohol screening tool in a UK dental setting to identify patients' alcohol consumption. Community Dent Health 2015; 32(4):196-8.

28. Roked Z, Moore S, Shepherd J. Feasibility of alcohol misuse screening and treatment in the dental setting. Lancet 2015; 385 Suppl:S84.

29. Ntouva A, Porter J, Crawford MJ, Britton A, Gratus C, Newton T, et al. Assessing the feasibility of screening and providing brief advice for alcohol misuse in general dental practice: a clustered randomised control trial protocol for the DART study. BMJ Open 2015; 5(10):e008586.

30. Alzahrani SG, Sheiham A, Tsakos G, Watt RG. Psychosocial determinants of clustering health-compromising behaviors among Saudi male adolescents. Int J Pediatr Adolesc Med 2017; 4(1):26-32.

31. Singh A, Rouxel P, Watt RG, Tsakos G. Social inequalities in clustering of oral health related behaviors in a national sample of British adults. Prev Med 2013; 57(2):102-6.

32. Myint PK, Luben RN, Wareham NJ, Bingham SA, Khaw KT. Combined effect of health behaviours and risk of first ever stroke in 20,040 men and women over 11 years' follow-up in Norfolk cohort of European Prospective Investigation of Cancer (EPIC Norfolk): Prospective population study. BMJ 2009; 338: b349.

33. Blot WJ, McLaughlin JK, Winn DM, Austin DF, Greenberg RS, Preston-Martin S, et al. Smoking and Drinking in Relation to Oral and Pharyngeal Cancer. Cancer Res 1988; 48:3282-7. 
Table 1: Audit-C questions and scoring system

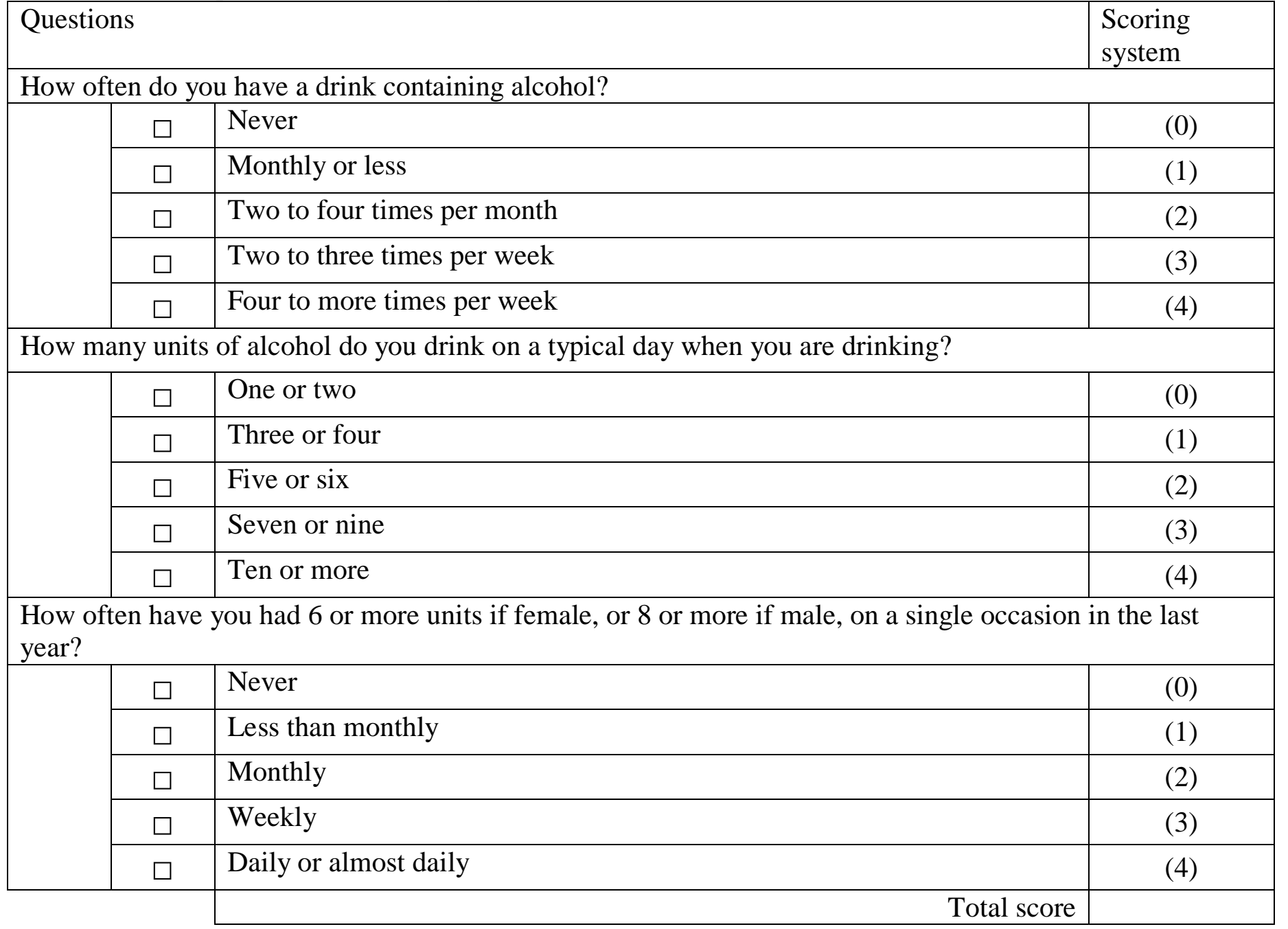


Table 2: Descriptive characteristics of the sample $(n=557)$

\begin{tabular}{|lll|}
\hline Variable & Total (N) & Prevalence (\%) \\
\hline Sex & & \\
Male & 232 & 42.0 \\
Female & 320 & 57.9 \\
\hline Age & & \\
18 to 34 years & 172 & 31.2 \\
35 to 49 years & 166 & 30.0 \\
50 and over & 214 & 38.8 \\
\hline Fruit and vegetable consumption & & \\
Never & 13 & 2.4 \\
Less than once a month & 12 & 2.2 \\
Once a month & 20 & 3.6 \\
Weekly & 240 & 43.5 \\
Daily & 267 & 48.4 \\
\hline Red meat consumption & & \\
Never & 47 & 8.5 \\
Less than once a month & 33 & 6.0 \\
Once a month & 53 & 9.6 \\
Weekly & 349 & 63.2 \\
Daily & 70 & 12.7 \\
\hline Smoking status & & \\
Current smoker & 134 & 24.3 \\
Ex-smoker & 138 & 25.0 \\
Never smoked & 280 & 50.7 \\
\hline Smoking frequency* & & \\
Less than daily & 22 & 15.8 \\
Daily & 117 & 54.2 \\
\hline AUDIT-C overall score & & 45.8 \\
Negative & 299 & \\
Positive & 253 & \\
\hline Curent smokers & & \\
\hline
\end{tabular}

*Current smokers only 
Table 3: Association between audit-c overall score and variables of interest $(n=552)$

\begin{tabular}{|c|c|c|c|}
\hline & \multicolumn{3}{|c|}{ Positive audit-c score ( $\geq 5$ points) } \\
\hline & $\mathbf{N}(\%)$ & $\begin{array}{l}\text { Odds Ratio (95\% } \\
\text { CI) }\end{array}$ & p-value \\
\hline \multicolumn{4}{|l|}{ Sex } \\
\hline Male & $139(59.9)$ & 1 & $<0.001$ \\
\hline Female & $114(35.6)$ & $0.4(0.3-0.5)$ & \\
\hline \multicolumn{4}{|l|}{ Age } \\
\hline 18 to 34 years & $104(60.5)$ & 1 & $<0.001$ \\
\hline 35 to 49 years & $76(45.8)$ & $0.5(0.4-0.9)$ & \\
\hline 50 and over & $73(34.1)$ & $0.3(0.2-0.5)$ & \\
\hline \multicolumn{4}{|l|}{ Fruit and vegetable consumption } \\
\hline Never & $5(38.5)$ & 1 & 0.21 \\
\hline Less than once a month & $5(41.7)$ & $1.1(0.2-5.8)$ & \\
\hline Once a month & $9(45.0)$ & $2.3(0.3-5.6)$ & \\
\hline Weekly & $128(53.3)$ & $1.8(0.8-5.8)$ & \\
\hline Daily & $106(39.7)$ & $1.1(0.3-3.3)$ & \\
\hline \multicolumn{4}{|l|}{ Red meat consumption } \\
\hline Never & $16(34.0)$ & 1 & $<0.001$ \\
\hline Less than once a month & $7(21.2)$ & $0.5(0.2-1.5)$ & \\
\hline Once a month & $15(28.3)$ & $0.8(0.3-2.8)$ & \\
\hline Weekly & $183(52.4)$ & $2.1(1.1-4.0)$ & \\
\hline Daily & $32(45.7)$ & $1.6(0.8-3.5)$ & \\
\hline \multicolumn{4}{|l|}{ Smoking status } \\
\hline Current smoker & $74(55.2)$ & 1 & $<0.001$ \\
\hline Ex-smoker & $71(51.4)$ & $0.9(0.5-1.4)$ & \\
\hline Never smoked & $108(38.6)$ & $0.5(0.3-0.8)$ & \\
\hline \multicolumn{4}{|l|}{ Smoking frequency* } \\
\hline Less than daily & $12(54.6)$ & 1 & 0.95 \\
\hline Daily & $63(53.9)$ & $0.9(0.4-2.4)$ & \\
\hline
\end{tabular}

*Current smokers only 


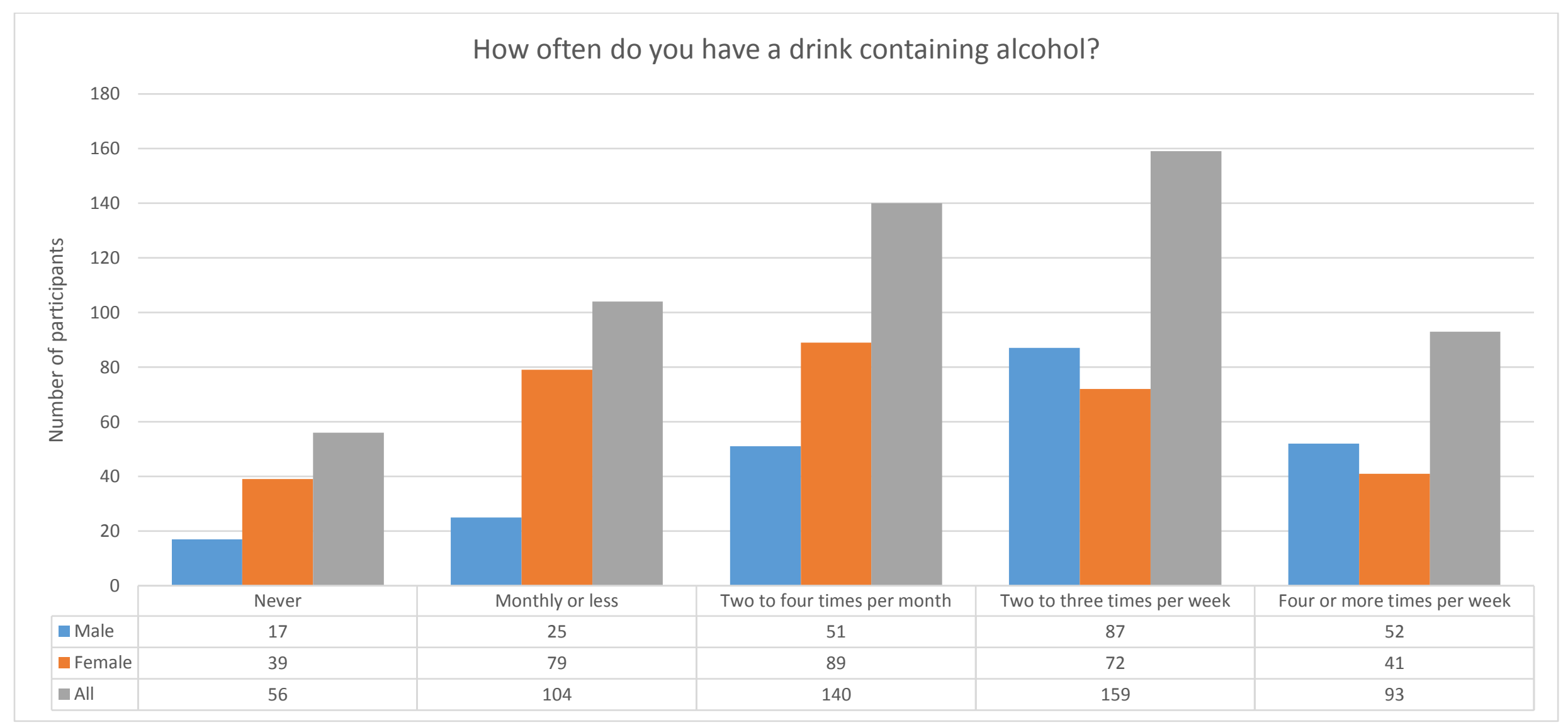

Figures 1: AUDIT-C question 1 responses by sex. 


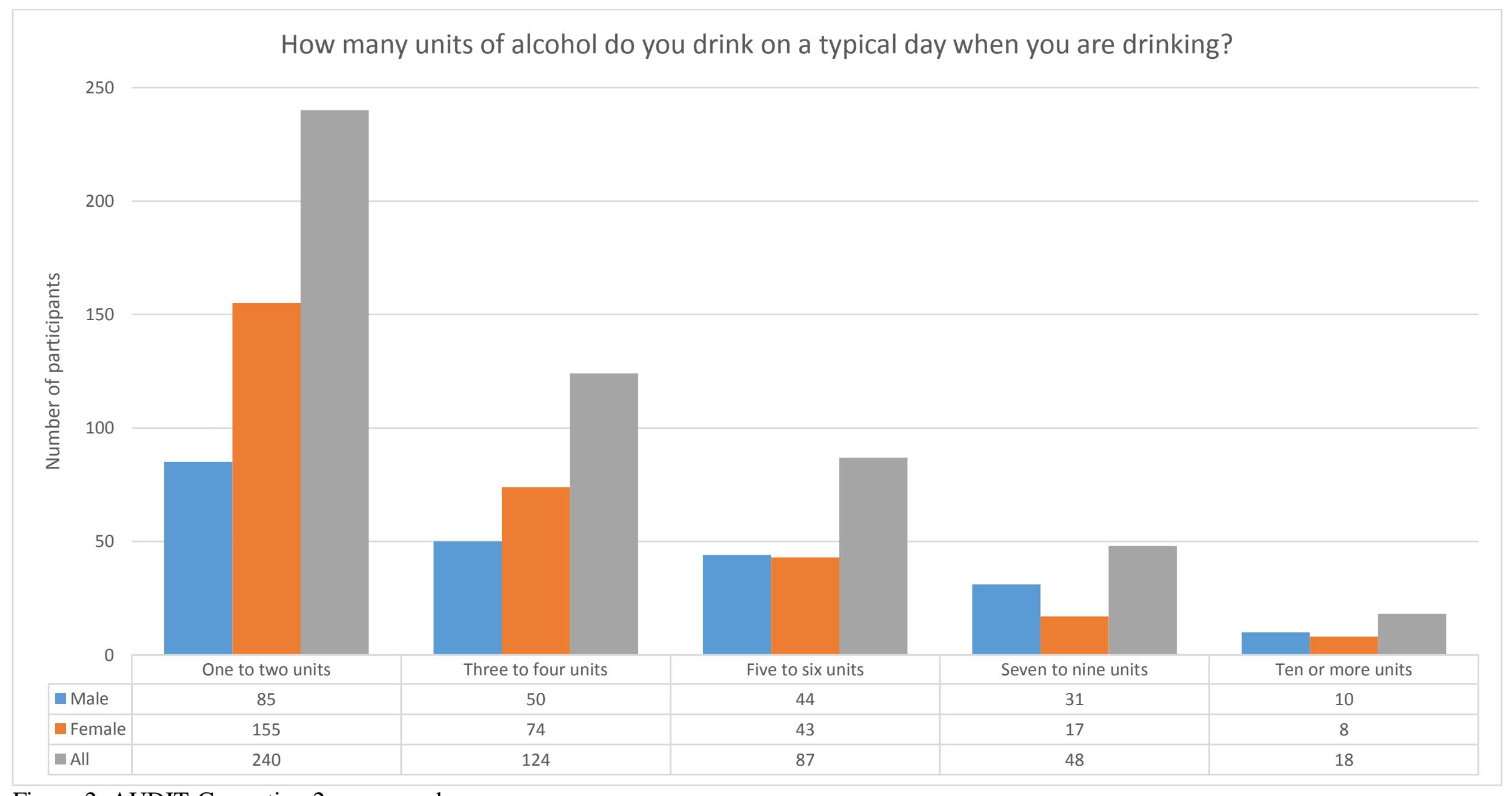

Figure 2: AUDIT-C question 2 responses by sex. 


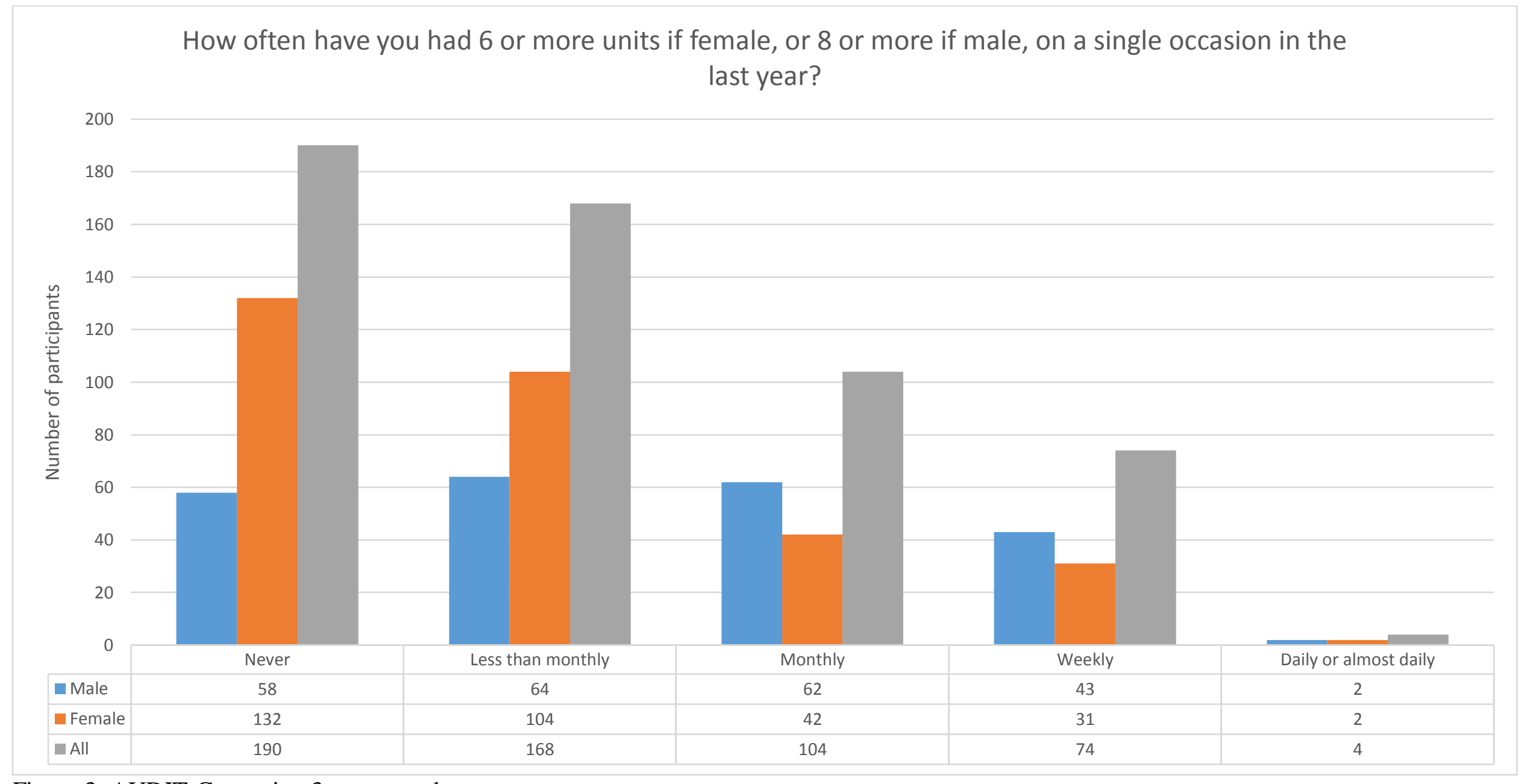

Figure 3: AUDIT-C question 3 responses by sex. 